Abbreviated Key Title: Sch J Dent Sci

ISSN 2394-4951 (Print) | ISSN 2394-496X (Online)

Journal homepage: http://saspjournals.com/sjds

\title{
Tooth in Infratemporal Fossa
}

Chaitanya Kumar. $\mathrm{N}^{1^{*}}$, Sridhar Reddy Kanubaddy ${ }^{2}$, Ramesh Babu Vaka ${ }^{3}$

${ }^{1}$ Post Graduate Student, ${ }^{2,3}$ Professor, Department of Oral and Maxillofacial Surgery, Narayana Dental College and Hospital, Nellore, Andhra Pradesh, India

DOI: $10.36347 /$ sjds.2020.v07i01.001

| Received: 18.12.2019 | Accepted: 25.12.2019 | Published: 10.01.2020

*Corresponding author: Chaitanya Kumar. N

Maxillary molars are usually displaced during forceful extraction into the maxillary sinus and rarely into the infratemporal fossa. Foreign bodies displaced into infratemporal fossa are challenging to manage due to its anatomical location and adjacent vital structures. The most commonly associated complications due to displaced foreign bodies into infratemporal fossa are haemorrhage, infection, pain, swelling, trismus etc. This article presents a rare case of accidentally displaced maxillary right third molar root stump into the adjacent infratemporal fossa during the extraction, which was diagnosed based on clinical and radiological examinations. There are various surgical modalities for removal of displaced root stump.

Keywords: Molar root stump, infratemporal fossa, surgical removal.

Copyright @ 2020: This is an open-access article distributed under the terms of the Creative Commons Attribution license which permits unrestricted use, distribution, and reproduction in any medium for non-commercial use (NonCommercial, or CC-BY-NC) provided the original author and source are credited.

\section{INTRODUCTION}

Foreign bodies encountered in maxillofacial regions are difficult to manage due to many factors like size of the object, difficult access and relationship of vital structures. The most common foreign bodies in maxillofacial regions are broken instrument tips, displaced teeth and root stumps or implants. While performing surgical procedures, they may get accidentally displaced into adjacent structures like maxillary sinus, lingual pouch, infratemporal fossa etc. Surgical removal of maxillary third molars is done routinely, which sometimes result in displacement into adjacent infratemporal fossa region [1].

Displaced foreign bodies may cause complications like excessive haemorrhage, infection, pain, swelling, trismus etc. Many factors are responsible for the displacement of foreign bodies into infratemporal fossa due to insufficient clinical and radiological examination, lack of surgical experience, anatomical alterations like thin cortical bony plate distal to maxillary third molars and angulation of teeth [2].

This clinical case report describes the location and surgical removal of maxillary third molar root stump, through intraoral approach, which was accidentally displaced into the infratemporal fossa.

\section{CASE REPORT}

A 35-year-old female patient reported to the department with a chief complaint of pain and occasional pus discharge from the right maxillary third molar region. Patient medical history was unremarkable. Clinical and radiological examinations revealed displaced maxillary third molar root stump into infratemporal fossa on the right side $(1 \mathrm{a}, 1 \mathrm{~b}, 1 \mathrm{c})$. 


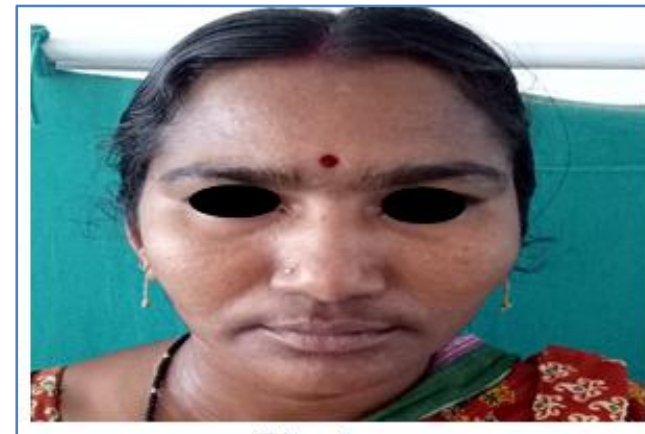

Fig: 1a

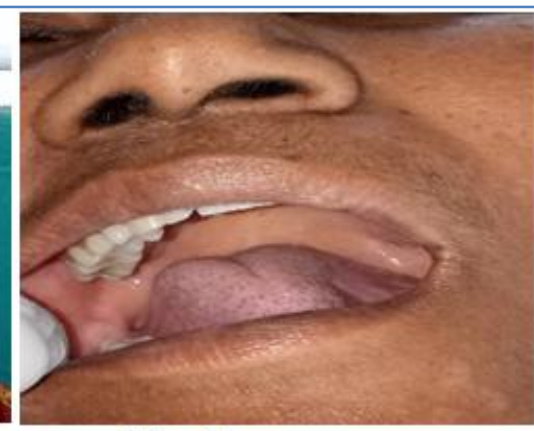

Fig: 1b

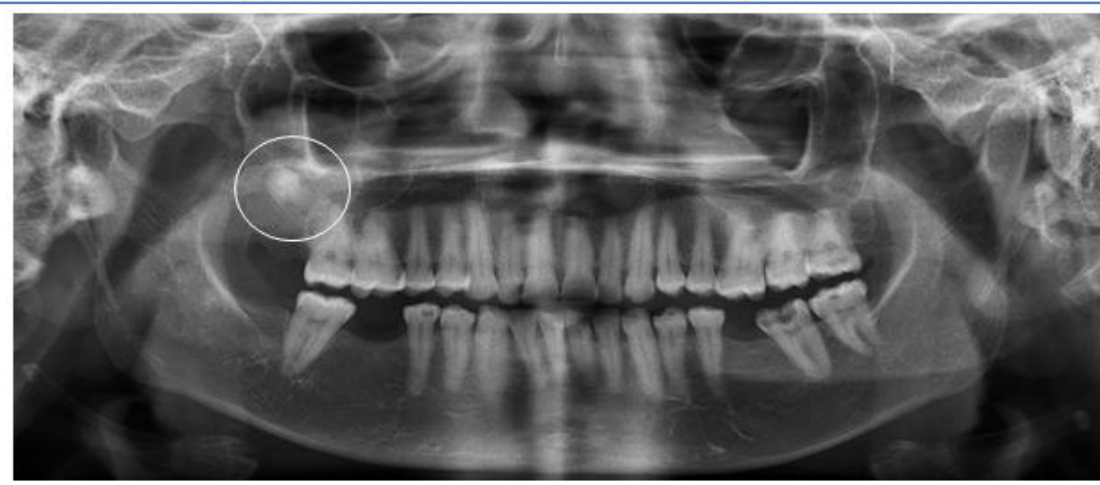

Fig-1c: Pre-operative OPG

\section{Surgical technique}

Under standard aseptic conditions, nasoendotracheal intubation done. Standard painting and draping was done. A vestibular incision was given from 15 to the distal aspect of maxillary tuberosity. A mucoperiosteal flap was raised, and from the distal aspect of tuberosity infratemporal fossa was accessed. Then third molar root stump was detected and removed. Haemostasis was achieved, and closure was done with vicryl (fig: $2 \mathrm{a}, 2 \mathrm{~b}, 2 \mathrm{c}$ ).
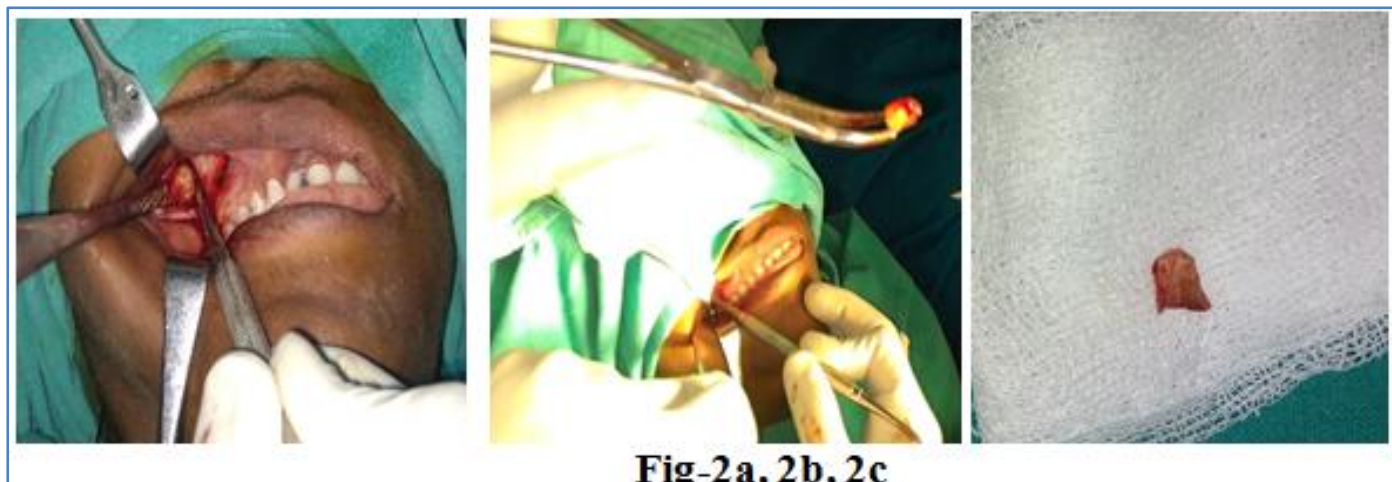

Fig-2a, 2b, 2c

\section{DISCUSSION}

The incidence of accidental displacement of tooth structures into adjacent areas is very rare. Usually, maxillary $3^{\text {rd }}$ molars are dislodged into infratemporal fossa while removing forcefully. There are many anatomical and vital structures present in the infratemporal fossa, which makes the surgical procedure very challenging. In general, oro-antral communication is the sign of displacement of a foreign body into the maxillary sinus, and limited mouth opening may be a sign of displacement of foreign body into the infratemporal fossa. Usually, teeth are displaced through periosteum into infratemporal fossa just adjacent to lateral pterygoid muscle. If the excessive force applied the tooth structure may get displaced into skull base results in higher risk for morbidity [2].

Foreign bodies in infratemporal fossa some time results in delayed complications like an aneurysm, foreign body granulomas, cellulitis, and discharging sinus tract.

Foreign body reaction would lead to fibrosis of musculature, which results in trismus and pain. The point of time for removal of displaced foreign bodies still remained controversial as per the review of the literature. According to one school of thought, it is better to retrieve the tooth immediately because of the possible risk of infection. On the other hand, some 
suggest waiting till 3-4 weeks for fibrosis to occur and tooth to get stabilized in a firm position [3].
Surgical access for the removal of tooth from the infratemporal fossa is challenging and has the possibility for morbidity due to the anatomical structures present [3].

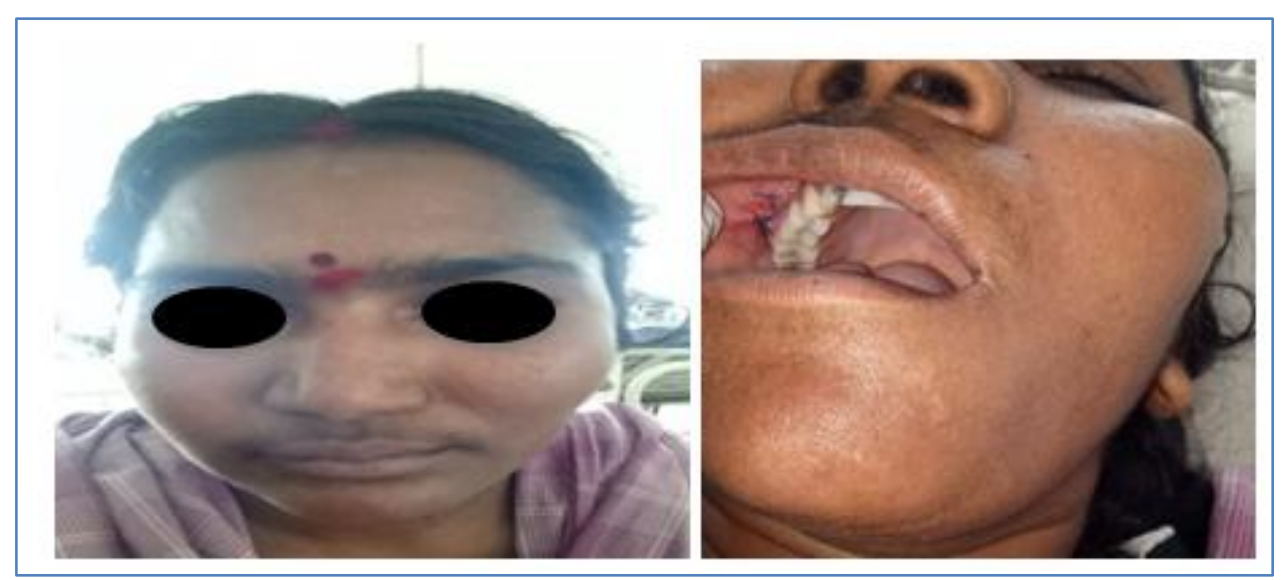

Fig-3a, 3b

\section{REFERENCES}

1. Selvi F, Cakarer S, Keskin C, Ozyuvaci H. Delayed removal of a maxillary third molar accidentally displaced into the infratemporal fossa. J Cranio Maxillofac Surg. 2011;22(4):1391-3.

2. Sverzut CE, Trivellato AE, Sverzut AT, DE Matos FP, Kato RB. Removal of a maxillary third molar accidentally displaced into the infratemporal fossa via intraoral approach under local anesthesia: report of a case. J Oral Maxillofac Surg. 2009;67(6):1316-20.

3. Shuker ST. Base-of-skull/infratemporal fossa shell fragment retrieval. J Oral Maxillofac Surg. 2010;68(11):2668-74. Shukser ST. Base-ofskull/infratemporal fossa shell fragment retrieval. J Oral Maxillofac Surg. 2010;68(11):2668-74. 